\title{
Chronic Carriage of Hepatitis B Virus at the University Teaching Hospital Yalgado Ouedraogo: Therapeutic Aspects and Outcome in a Cohort of HBeAg+ Outpatients
}

\author{
Eric Nagaonlé Somé ${ }^{1 *}$ (), Félicité W. Nana ${ }^{1}$, Bertrand I. Méda ${ }^{1}$, Moussa Son ${ }^{2}$, Drabo Maxime ${ }^{1}$, \\ Séni Kouanda ${ }^{1}$, Roger Sombié ${ }^{2}$ \\ ${ }^{1}$ Biomedical and Public Health Department, Institut de Recherche en Sciences de la Santé (IRSS), Ouagadougou, Burkina Faso \\ ${ }^{2}$ Gastro-Enterology Department, UFR des Sciences de la Santé, Université Joseph Ki-Zerbo, Ouagadougou, Burkina Faso \\ Email: ^eric.some@gmail.com, felynanaw@gmail.com, son_moussa@yahoo.fr,mdrabok@gmail.com,docsomb@gmail.com
}

How to cite this paper: Somé, E.N., Nana, F.W., Méda, B.I., Son, M., Maxime, D., Kouanda, S. and Sombié, R. (2021) Chronic Carriage of Hepatitis B Virus at the University Teaching Hospital Yalgado Ouedraogo: Therapeutic Aspects and Outcome in a Cohort of $\mathrm{HBeAg}+$ Outpatients. Open Journal of Epidemiology, 11, 245-255. https://doi.org/10.4236/ojepi.2021.113021

Received: January 27, 2021

Accepted: July 20, 2021

Published: July 23, 2021

Copyright $\odot 2021$ by author(s) and Scientific Research Publishing Inc. This work is licensed under the Creative Commons Attribution International License (CC BY 4.0).

http://creativecommons.org/licenses/by/4.0/

(c) (i) Open Access

\begin{abstract}
Introduction: The HbsAg prevalence in Burkina Faso was 9.1\%. We aimed at describing the therapeutic features and the clinical outcome for the patients taking antiretroviral treatment. Materials and Methods: We implemented a cross-sectional study from January 1st, 2004 to December 31st, 2015. Patients aged more than 15 years with positive hepatitis B surface antigen for over six months and positive hepatitis B e-antigen were included. Results: We analyzed the data of 148 participants for a sex ratio of 3; sixty-three patients including 49 men $(77.8 \%)$ were on treatment. and $81.5 \%$ had inflammatory activity greater than one. Under tenofovir, the normalization of ALT was observed in 42 (84\%) patients while HBV-DNA became undetectable in 24/33 patients. HBeAg negativation was observed in 16/25 (64\%) patients after seven years of treatment. With lamivudine, $2 / 9$ patients had a complete virologic response and six had a normalization of their ALT. Two and 9 patients lost HBeAg after 7 and 9 years of treatment, respectively. Overall $63 \%$ and $27 \%$ of the patients were in the high or low-adherence group, respectively. In the low-adherence group, all patients had normal or abnormal ALT, but detectable HBV DNA. Ten patients taking lamivudine developed resistance including primary resistance in three patients. No resistance has been observed with tenofovir. Conclusion: The management of the viral hepatitis B includes often a long follow up period without any medication. When antiviral is indicated, the adherence to the treatment is crucial to a long-term control of the virus. In our setting, the low purchase power of the patients may jeopardize their therapeutic future and there is a need to support this group of patients with free-of-charge medicines as it is provided for the HIV infected people.
\end{abstract}




\section{Keywords}

Viral Hepatitis B, HBe Antigen, Antiretroviral Therapy, Adherence, Resistance

\section{Introduction}

Chronic viral hepatitis B refers to an inflammatory disease of the liver caused by the hepatitis B virus (HBV) which has been active for more than six months. It is a major public health problem, since the prevalence of chronic HBV carriage, is over $7 \%$ or even $20 \%$ in sub-Saharan African countries [1]. According to the World Health Organization (WHO) in 2015, around 257 million people suffered from chronic hepatitis B worldwide with a higher frequency in African countries and more than 800,000 people died from it [2] [3].

Despite the effective vaccine recommended by WHO against hepatitis B since 1991 and introduced in the Expanded Program of Immunization in Burkina Faso since 2006, the number of patients infected with HBV still remains high. Burkina Faso is ranked as a highly endemic area [3] with a prevalence of the hepatitis B surface antigen (HBsAg) estimated at 9.1\% [4]. This situation is explained by the fact that in sub-Saharan Africa, the HBV infection, most often acquired at birth, promotes chronic carriage due to the immunotolerance reaction of the body [5].

The HBV replication may be due to the wild virus excreting the hepatitis $B$ e-antigen ( $\mathrm{HBeAg}$ ) or the preC/C mutant preventing the excretion of $\mathrm{HBeAg}$. Although $\mathrm{HBeAg}$ is associated with high contagiousness, it is the detection and quantification of HBV-DNA that remains the best test for viral replication. This phase of viral replication, when uncontrolled by the body, could progress to cirrhosis or primary liver cancer. It should prompt doctors to discuss the indication of an antiviral treatment. In the management of chronic viral hepatitis $B$ (VHB) in HbeAg+ patients, whether or not under medication, the objective is to obtain the HBe seroconversion which most often allows the control of the infection, especially by the immune system. We collected the data in the context of a medical doctorate thesis with the general objective to gather baseline data in an HbsAg positive cohort, for future follow-up. The decision to publish the data was made lately in order to feed the global knowledge on the topic and mainly to allow comparison with other future studies in Sub-Saharan Africa. The aim of the current paper was to describe the therapeutic aspects and the treatment's outcomes in HbeAg+ patients in the hepato-gastroenterology department of the University Teaching Hospital Yalgado Ouédraogo (CHU-YO).

\section{Materials and Methods}

We implemented at the hepato-gastroenterology department of the University Teaching Hospital Yalgado Ouédraogo (CHU-YO), a cross-sectional descriptive 
study with a retrospective data collection from January 1st, 2004 to December 31st, 2015. The study population consisted of 1) HbsAg+ patients screened by the national blood transfusion center and referred to the hepato-gastroenterology department of CHU-YO for treatment and 2) chronic VHB HbeAg+ patients who were followed-up as outpatients in the same department. Patients aged more than 15 years with HbsAg+ for over six months and HbeAg+ were included. Patients who had a decompensated cirrhosis or a primary liver cancer were excluded. Data were collected using a data collection form.

\subsection{Study Overview}

Once recruited, the patients' paper medical files were used to collect information on civil status, weight, height, telephone number, lifestyle, risk factors and clinical, biological, radiological and histological data. A monthly, quarterly and bi-annual check of the transaminases (alanine aminotransferase (ALT)) was carried out. Tests for HBsAg, $\mathrm{HBeAg}$ and $\mathrm{HBV}$-DNA were performed every six months or every year. HBsAg was detected using the rapid diagnostic test (the Determine TM Abbott) or the Elisa method (Vidas ${ }^{\circledast}$ ). The quantification of HBsAg was carried out by the HBs Ag II quant II Cobas method: $0.05 \mathrm{IU} / \mathrm{mL}$ to 52,000 IU/mL ( $\log 10: 2.11)$ and the quantification of HBV-DNA by a real-time PCR (Roche Cobas Taq Man, sensitivity threshold $20 \mathrm{IU} / \mathrm{mL}$ ). An abdominal ultrasound and/or an abdominal CT scan were performed annually in the absence of cirrhosis and every six months otherwise. Liver activity and fibrosis were assessed by a liver biopsy or a liver fibrosis blood test using a FibroMeter ${ }^{\circledR}$.

Antiviral treatment was indicated in patients with activity and/or fibrosis $\geq 2$. When ALT concentration was twice the superior margin of the range in a patient with detectable viral load, antiviral treatment was also initiated regardless of the activity or the fibrosis. A family history of primary liver cancer and the ability to adhere to the treatment were also considered before the treatment initiation. Using a questionnaire and a visual analogue scale (VAS) from 0 to 10 (0: the patient stopped any medication and 10: the patient takes regularly its drugs at the right time), the adherence to the treatment was assessed over the last three months. Three categories were defined with regard to the adherence: The high adherence group (no missed dose, VAS score of 10), moderate adherence group (overall good adherence over the last three months, VAS score from 8.1 to 9.9), and low or non-adherence group (seldom drug intake or treatment completely stopped over the last three months, VAS score $\leq 8$ ). The statistical analysis was carried out using Epi Info software.

\subsection{Operational Definitions, Biochemical and Virological Profiles}

- HBV chronic carrier: a subject whose HBsAg test is positive for more than 6 months and the anti-hepatitis B core antibodies (anti-HbcAb; total IgG) test is positive.

- Chronic active hepatitis: a chronic hepatitis B carrier whose ALT quantifica- 
tion is twice above the superior threshold of the normal range of values, continuously or in a fluctuating manner and the HBV-DNA is detectable.

- Chronic inactive carrier: a chronic hepatitis B carrier whose ALT quantification is still within the normal range and the HBV-DNA is less than 2000 $\mathrm{IU} / \mathrm{ml}$.

- Duration of HBsAg carriage: this is the time interval between the date of HbsAg detection and the end of our study.

- Primary non-response: was defined as a $<1$-log drop after 3 months of treatment as per EASL's (European Association for the Study of the Liver) definition [6].

- Complete virological response: decrease of serum HBV DNA to PCR-undetectable levels after 1 year of treatment.

- Resistance: It corresponds to the rise in serum HBV DNA levels of at least 1 - $\log _{10} \mathrm{IU} / \mathrm{mL}$ compared with the lowest value during therapy (nadir value), in 2 consecutive samples 1 month apart, in patients who have previously responded and have a good treatment compliance [7].

- Primary resistance: when viral DNA remains unchanged after three months of treatment.

- Immunotolerance: a patient whose HBV-DNA > 20,000 IU/ml with its ALT still within the normal range.

- HBs seroconversion: it is the loss of the HBsAg with or without the detection of the Anti-HBsAb and the Anti-HBcAb is negative.

- HBe seroconversion: it is the loss of the HBeAg with or without the detection of the anti-HBeAb.

We categorized five biochemical and virological profiles:

- First profile: HBV-DNA < $2000 \mathrm{IU} / \mathrm{ml}$ and ALT still within the normal range (inactive carriage);

- Second profile: a high HBV-DNA > 20,000 IU/ml and ALT consistently above the normal range;

- Third profile: a fluctuating viral replication and ALT values without any normalization window;

- Fourth profile: a succession of periods of elevated ALT followed by spontaneous normalization and a fluctuating viral replication;

- Fifth profile: the immunotolerance phase with an HBV-DNA > 20,000 IU/ml and ALT consistently within the normal range.

\section{Results}

From January 1st, 2004 to December 31st, 2015, we recruited a total of 1264 patients with chronic VHB including 148 (11.7\%) carriers of HBeAg.

\subsection{Sociodemographic Characteristics}

The sample included 148 participants for a sex ratio of $3 ; 69 \%$ of the participants were $\leq 34$ years old. There were $66(44.6 \%)$ students, and $63(29 \%)$ workers in the 
formal public or private sectors. Singles or widows represented 93 (62.8\%) participants. Blood donation 66 (44.6\%) and routine check-up 43 (29\%) were the most frequent circumstances for diagnosing HBV infection.

Abdominal ultrasound was normal in $126(85.1 \%)$ patients. A fatty or dysmorphic liver was observed in $9(6.1 \%)$ and 7 (4.7\%) cases, respectively.

The hepatic fibrosis blood test showed an activity $\mathrm{A} \geq 2$ in 25 (83.3\%) patients and fibrosis $\mathrm{F} \geq 2$ in $23(76.7 \%)$ patients. The liver biopsy was performed in 8 (5.4\%) patients; 2 patients showed no inflammatory activity (A0); 5 had a minimal activity (A1). A portal fibrosis without septa (F1) was found in three patients and three others had portal fibrosis with some septa (F2).

\subsection{Therapeutic Features}

Sixty-three patients including 49 (77.8\%) men were on treatment. The remaining participants were not taking any drug either because antiviral drugs were not yet indicated for their disease status, or because they were not able to complete the biological investigations to make the decision to start a treatment. The mean age for those under treatment was 33.8 years (range: 21 to 62). The most represented occupations were pupils or students 45 (30.2\%), civil servants $42(28.6 \%)$ and private employees $33(22.2 \%)$. Fibrometer testing was performed in 27 patients and $14(81.5 \%)$ had inflammatory activity greater than one.

Tenofovir was administered to $50(79.4 \%)$ patients and lamivudine to 9 (14.3\%) patients (Figure 1).

The duration of the treatments with tenofovir ranged between 1 and 9 years. Normalization of ALT was observed in $42(84 \%)$ patients while HBV-DNA became undetectable in 24/33 patients. HBeAg negativation was observed in 16 (64\%) patients after seven years of treatment.

Of the nine patients on Lamivudine, two had a complete virologic response and six had a normalization of their ALT (Table 1). Two and 9 patients lost HBeAg after 7 and 9 years of treatment, respectively. No case of HBsAg seroconversion has been observed under lamivudine treatment.

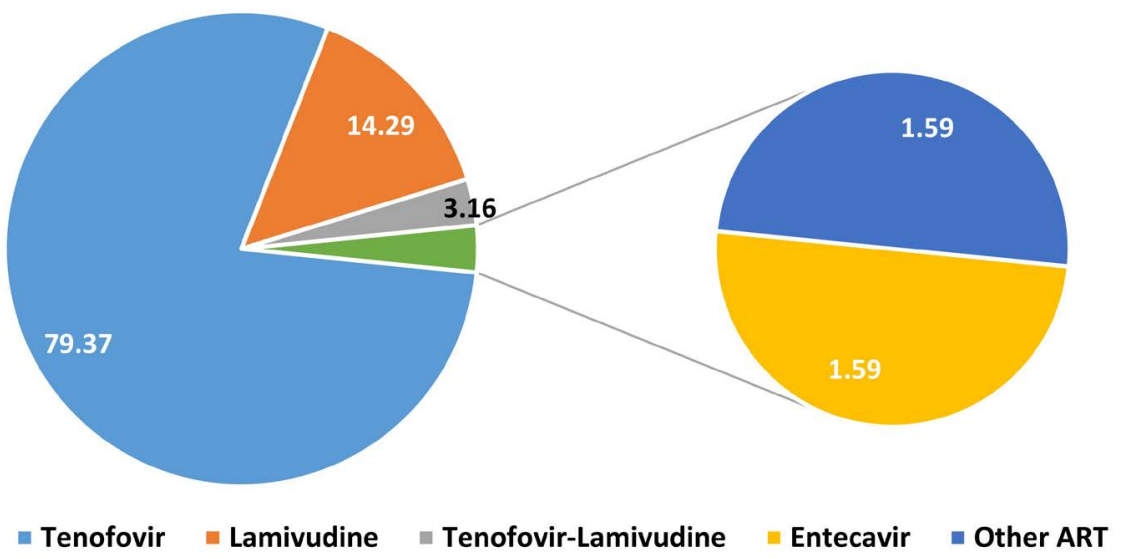

Figure 1. Distribution of patients according to the antiretroviral treatment. ${ }^{\star}$ Anti-retroviral treatment. 
Table 1. Changes in ALT concentrations and HBV DNA load according to the treatment duration.

\begin{tabular}{|c|c|c|c|c|c|c|c|c|}
\hline \multirow{2}{*}{$\begin{array}{c}\text { Treatment } \\
\text { duration } \\
\text { (year) }\end{array}$} & \multicolumn{4}{|c|}{ Tenofovir treatment } & \multicolumn{4}{|c|}{ Lamivudine treatment } \\
\hline & $\begin{array}{c}\text { Normal } \\
\text { ALT }\end{array}$ & $\begin{array}{c}\text { Abnormal } \\
\text { ALT }\end{array}$ & $\begin{array}{l}\text { Undetectable } \\
\text { Viral DNA }\end{array}$ & $\begin{array}{l}\text { Detectable } \\
\text { Viral DNA }\end{array}$ & $\begin{array}{c}\text { Normal } \\
\text { ALT }\end{array}$ & $\begin{array}{l}\text { Abnormal } \\
\text { ALT }\end{array}$ & $\begin{array}{c}\text { Undetectable } \\
\text { Viral DNA }\end{array}$ & $\begin{array}{l}\text { Detectable } \\
\text { Viral DNA }\end{array}$ \\
\hline$<1$ & 8 & 2 & 1 & 3 & & & & \\
\hline 1 & 2 & 0 & 2 & 0 & & & & \\
\hline 2 & 5 & 0 & 1 & 1 & & & & \\
\hline 3 & 6 & 1 & 4 & 1 & & & & \\
\hline 4 & 5 & 1 & 4 & 0 & 1 & 0 & 0 & 1 \\
\hline 5 & 2 & 2 & 2 & 0 & 0 & 0 & 0 & 0 \\
\hline 6 & 11 & 2 & 7 & 3 & 2 & 1 & 0 & 0 \\
\hline 7 & 2 & 1 & 2 & 1 & 1 & 1 & 1 & 1 \\
\hline 8 & 1 & 0 & 1 & 0 & 1 & 1 & 1 & 0 \\
\hline 9 & 8 & 2 & 1 & 3 & 1 & 0 & 0 & 0 \\
\hline Total & 42 & 9 & 24 & 9 & 6 & 3 & 2 & 2 \\
\hline
\end{tabular}

\subsection{Therapeutic Adherence}

Overall $40(63 \%)$ patients were highly adherent, 17 (27\%) were in the low adherence group and $6(10 \%)$ were moderately adherent (Figure 2$)$. According to the occupation, religious people and housewives were the most adherent (Figure 3), followed by the private sector employees, the civil servants and the students/pupils and tradespeoples at the same proportion of $50 \%$. The highly adherent group among farmers represented $33 \%$. The 15 - 24 age group was the least adherent (20\% of the group was adherent) while the group of the 45 - 54 years old was highly (100\%) adherent. The other age groups (25 - 34; 35 - 44 and 55 64 ) had an adherence level of over $60 \%$. After three years of treatment, $72.2 \%$ of the patients were adherent against $76.3 \%$ at 5 years; $11 \%$ and $13.2 \%$ were moderately adherent at respectively 3 and 5 years, and $16.8 \%$ and $10.5 \%$ were not at all adherent still at the same time period. Among highly or moderately adherent patients, ALT were normal and viral DNA undetectable in $76.5 \%$ and $33.3 \%$ of cases, respectively. In the low adherent group, all patients had normal or abnormal transaminases, but detectable HBV DNA.

\subsection{Resistance to Treatment and Disease Progression}

Lamivudine was initiated in 21 patients, 9 of whom were still under the molecule at the time of our study. Ten had developed resistance including primary resistance in three patients. In two patients, lamivudine was switched to tenofovir after 4 years of treatment to prevent the development of resistance. The resistance in one year was $14.3 \%$ and $47.6 \%$ in 5 years of treatment, meaning a mean increase of $12 \%$ per year. No resistance has been observed with tenofovir. Progressively, 4/41 patients with active VHB had become stable (inactive carriage with normal ALTs and HBV-DNA < $2000 \mathrm{IU} / \mathrm{mL}$ ) over a follow-up period of 1 to 6 


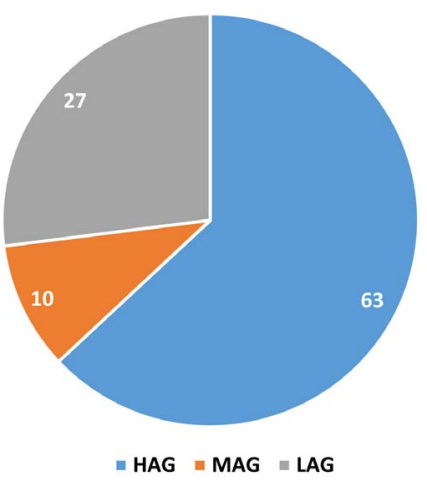

Figure 2. Distribution of the patients according to the adherence category. HAG: high adherence group; MAG: moderate adherence group; LAG: low adherence group.

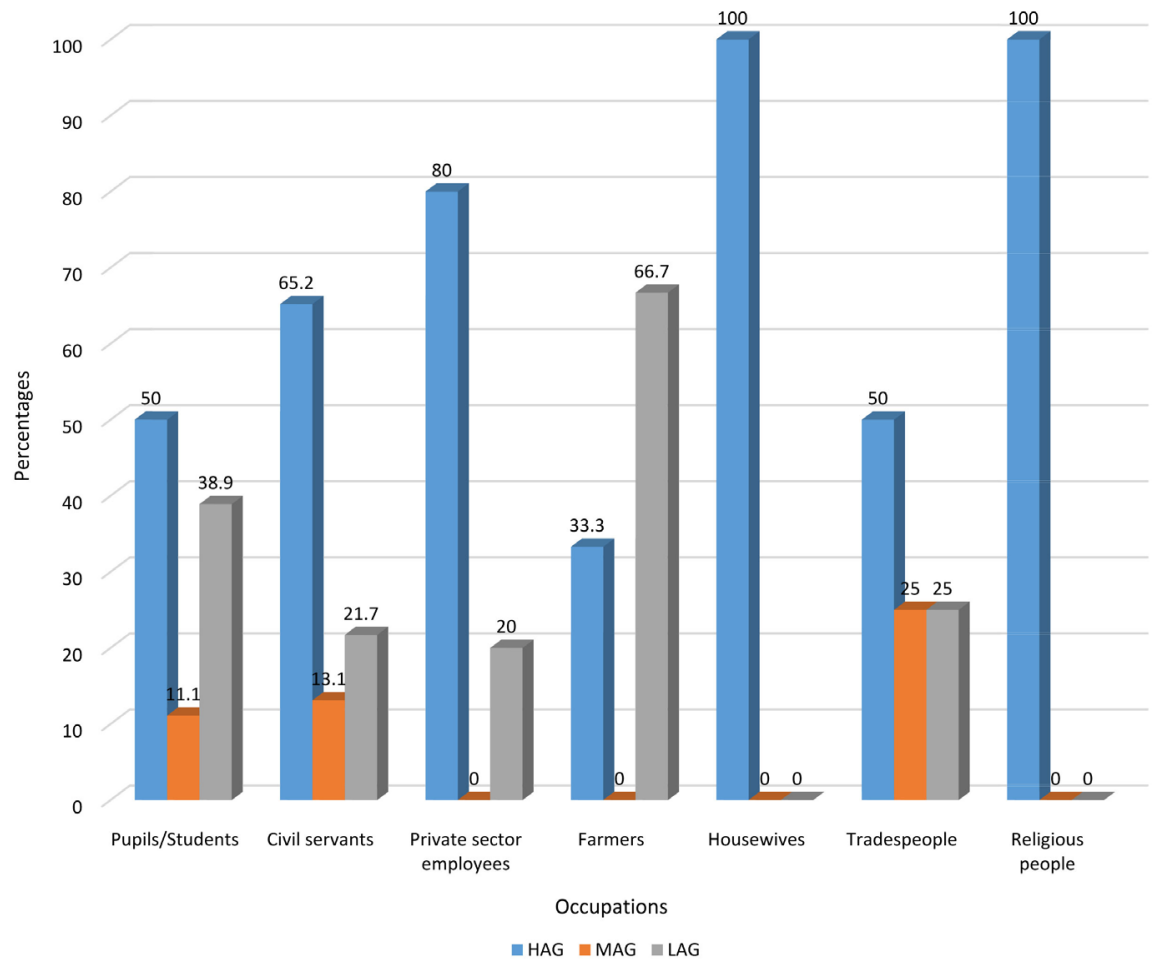

Figure 3. Distribution of the patients according to the occupation and the adherence category. HAG: high adherence group; MAG: moderate adherence group; LAG: low adherence group.

years. HBeAg seroconversion was observed in two inactive carriers. HBeAg negativation was found in 9 patients (22\% of active carriers). Thirty-seven (90.2\%) patients with chronic active hepatitis were observed among the untreated population.

\section{Discussion}

\subsection{Sociodemographic Characteristics}

With a sex ratio $=3$, our results were comparable to those of other studies on the topic [8] [9] and clearly indicated that VHB became chronic more often in men 
than in women.

The mean age was 33 years and $85.8 \%$ of the participants were younger than 40. Bougouma and Khelifa found a mean age of 37.5 and 35 years, respectively among patients with positive HBeAg. Other studies found a mean age as low as 33 years [8] [10]. In the literature [11], patients with $\mathrm{HBeAg}$ are generally $<40$ years.

Pupils and students represented $45 \%$ of our sample, followed by civil servants and private sector employees (Figure 3 ). These groups used to be the target population of blood collection campaigns and/or the yearly health check-up as part of occupational medical visits. The education level of these groups was undoubtedly also a contributing factor to the high rate of representativeness

A family history of HBsAg carriage, cirrhosis or primary liver cancer was found in $12.8 \%$ of cases. The weakness of HBV screening in the general population may be the explanation here.

\subsection{Therapeutic Features}

The virological response to tenofovir was complete in $72.7 \%$ of cases. This rate is comparable to the virological response reported in the literature, which fluctuates between $72 \%$ and $76 \%$ [12] [13]. Furthermore, the ALT normalized in $84 \%$ of our patients taking tenofovir over a period of 1 to 6 years (Table 1) versus $69 \%$ in the literature [12] for a follow-up of one year.

According to the defined categories of therapeutic adherence, 40 (63\%) patients were highly adherent; $6(10 \%)$ patients were moderately adherent and 17 (27\%) patients were in the low-adherence group (Figure 2). In other countries, the high adherence level was similar. Besides, $32 \%$ and $7 \%$ of cases were in the moderate or low-adherence group [14] [15]. The high proportion of low-adherent patients in our survey could be explained by the skipped doses because of the lack of habit at the beginning of the treatment and the consecutive forgetfulness, a lack of money to supply drugs [16], the drugs shortage at the level of the unique national supplier of antiretroviral drugs including lamivudine and tenofovir.

Adherence is the main factor associated with the viral suppression and its maintenance over time; conversely, non-adherence is a risk factor for the emergence of resistant strains [17]. The 15- to 24-year age group was $80 \%$ low-adherent. The adherence to a long-term medication is usually a challenge in young subjects. Generally speaking and in relation to the HBV infection, there is no need to rush to initiate antiviral treatment for young people; rather, the emphasis should be put on the clinical follow-up.

The rates of biochemical and virological responses decreased with decreasing adherence [15] [16] [17]. Adherence to treatment would therefore be a predictor of the viral suppression and normalization of transaminases. Consequently, the biochemical and virological responses were better in the context of high adherence. 
In our study, $47.6 \%$ of the participants under lamivudine molecule developed a resistant strain of the virus by five years of treatment. In the literature, the frequency of the resistance to lamivudine is $70 \%$ by five years [12]. In our setting, only a small number of patients could afford to get a laboratory viral load assessment and the proportion of resistance to lamivudine may have been underestimated. No case of resistance to tenofovir has been detected in our study and this finding is confirmed by other studies that did find no or very rare cases of resistance to tenofovir [7] [13].

\subsection{Study Limits}

A significant number of patients participating in the study, lacked laboratory and ultrasound data. Financial reason seemed to be behind this, as the study was designed to analyze routine data. We therefore believe that the description given in this work does not present the whole reality of our cohort. However, what is observed gives us a fairly objective picture of the evolution of a regularly monitored VHB patient.

\section{Conclusion}

The viral hepatitis B is a silent infection with a small proportion of patients experiencing viral replication activity. The management includes often a long follow up period without any medication. When antiviral is indicated, the adherence to the treatment is crucial to a long-term control of the virus. In our setting, the virological and biochemical responses were in general better or similar to other cohorts'. However, the low purchase power of the patients may jeopardize their therapeutic future and there is a need to support this group of patients with free-of-charge medicines as it is provided for the HIV-infected people.

\section{Conflicts of Interest}

The authors declare no conflicts of interest regarding the publication of this paper.

\section{References}

[1] Schweitzer, A., et al. (2015) Estimations of Worldwide Prevalence of Chronic Hepatitis B Virus Infection: A Systematic Review of Data Published between 1965 and 2013. The Lancet, 386, 1546-1555. https://doi.org/10.1016/S0140-6736(15)61412-X

[2] World Health Organization (2017) Global Hepatitis Report. Global Hepatitis Programme, World Health Organization, Geneva, 83.

[3] World Health Organization (2017) Hepatitis B Vaccines: WHO Position Paper-July 2017. Weekly Epidemiological Record, 27, 369-392.

[4] Meda, N., et al. (2018) Hepatitis B and C Virus Seroprevalence, Burkina Faso: A Cross-Sectional Study. Bulletin of the World Health Organization, 96, 750-759. https://doi.org/10.2471/BLT.18.208603 
[5] Lee, H.A., et al. (2020) Extremely Low Risk of Hepatocellular Carcinoma Development in Patients with Chronic Hepatitis B in Immune-Tolerant Phase. Alimentary Pharmacology \& Therapeutics, 52, 196-204. https://doi.org/10.1111/apt.15741

[6] Yang, Y.J., et al. (2014) Assessment of Current Criteria for Primary Nonresponse in Chronic Hepatitis B Patients Receiving Entecavir Therapy. Hepatology, 59, 1303-1310. https://doi.org/10.1002/hep.26910

[7] Zoulim, F. and Locarnini, S. (2009) Hepatitis B Virus Resistance to Nucleos(t)ide Analogues. Gastroenterology, 137, 1593-1608 e1-2. https://doi.org/10.1053/j.gastro.2009.08.063

[8] Bermingham, S.L., et al. (2015) Cost-Effectiveness Analysis of Alternative Antiviral Strategies for the Treatment of HBeAg-Positive and HBeAg-Negative Chronic Hepatitis B in the United Kingdom. Value Health, 18, 800-809.

https://doi.org/10.1016/j.jval.2015.05.007

[9] Nita, M.E., et al. (2009) Patterns of Viral Load in Chronic Hepatitis B Patients in Brazil and Their Association with ALT Levels and HBeAg Status. Annals of Hepatology, 8,339-345. https://doi.org/10.1016/S1665-2681(19)31747-8

[10] Khelifa, F. and Thibault V. (2009) Characteristics of Hepatitis B Viral Strains in Chronic Carrier Patients from North-East Algeria. Pathologie Biologie (Paris), 57, 107-113. https://doi.org/10.1016/j.patbio.2008.07.031

[11] Kondili, L.A., et al. (2005) Clinical and Molecular Characterization of Chronic Hepatitis B in Albania: A Country That Is Still Highly Endemic for HBV Infection. Journal of Medical Virology, 75, 20-26. https://doi.org/10.1002/jmv.20231

[12] European Association for the Study of the Liver (2009) EASL Clinical Practice Guidelines: Management of Chronic Hepatitis B. Journal of Hepatology, 50, 227-242. https://doi.org/10.1016/j.jhep.2008.10.001

[13] Yao, G.B., et al. (2009) A 7-Year Study of Lamivudine Therapy for Hepatitis B Virus E Antigen-Positive Chronic Hepatitis B Patients in China. Journal of Digestive Diseases, 10, 131-137. https://doi.org/10.1111/j.1751-2980.2009.00375.x

[14] ANRS and AFEF (2014) Prise en charge des personnes infectées par les virus de l'hépatite B ou de l'hépatite C. EDK/Groupe EDP Sciences,. Paris, 527 p.

[15] Abu-Freha, N., et al. (2020) Adherence to Anti-Viral Treatment for Chronic Hepatitis B. Journal of Clinical Medicine, 9, 1922.

https://doi.org/10.3390/jcm9061922

[16] Xu, K., et al. (2018) Adherence and Perceived Barriers to Oral Antiviral Therapy for Chronic Hepatitis B. Global Health Action, 11, 1433987. https://doi.org/10.1080/16549716.2018.1433987

[17] Lieveld, F., et al. (2013) Patient Adherence to Antiviral Treatment for Chronic Hepatitis B and C: A Systematic Review. Annals of Hepatology, 12, 380-391. https://doi.org/10.1016/S1665-2681(19)31000-2 


\section{List of Abbreviations}

$\begin{array}{ll}\text { Abbreviation } & \text { Definition } \\ \text { ALT } & \text { Alanine amino Transferase } \\ \text { CHU-YO } & \text { Centre Hospitalo-Universitaire Yalgado Ouédraogo } \\ & \text { (University Teaching Hospital Yalgado Ouédraogo) } \\ \text { HBeAg } & \text { Hepatits B e-Antigen } \\ \text { HBsAg } & \text { Hepatitis B surface Antigen } \\ \text { HBV } & \text { Hepatitis B Virus } \\ \text { HIV } & \text { Human Immuno-deficiency Virus } \\ \text { IU } & \text { International Unit } \\ \text { VHB } & \text { Viral Hepatitis B } \\ \text { WHO } & \text { World Health Organization }\end{array}$

